\title{
Mechanical Testing for Three-Dimensional Motion Analysis Reliability
}

\author{
$\underline{\text { Authors }}$ \\ Emily Miller, MS; Motion Analysis Laboratory, Mayo Clinic, Rochester, MN \\ Kenton Kaufman, Ph.D., P.E., Biomechanics-Motion Analysis Laboratory, Mayo Clinic, \\ Rochester, MN \\ Trevor Kingsbury, MS; Naval Medical Center San Diego, CA \\ Erik Wolf, PhD; Walter Reed National Military Medical Center, Bethesda, MD \\ Jason Wilken, PhD, PT; Center for the Intrepid, Brooke Army Medical Center, JBSA Fort Sam \\ Houston, TX \\ Marilynn Wyatt, MA, PT; Naval Medical Center San Diego, CA \\ Corresponding author: $\quad$ Kenton R. Kaufman, Ph.D., P.E. \\ Biomechanics-Motion Analysis Laboratory \\ Charlton North L-110L \\ 200 First Street SW \\ Rochester, MN 55905 \\ Email: kaufman.kenton@mayo.edu
}

Keywords: Gait, Motion Analysis, Reliability, Motion Capture System Validation

Source of support: DOD 731743-1, DOD Defense Health Programs/Center for Rehabilitative Sciences Research, Grant \# HU0001-11-1-0004

(C) 2016. This manuscript version is made available under the Elsevier user license 


\begin{abstract}
The purpose of this study was to use simple mechanical tests to evaluate the reliability of threedimensional motion analysis systems and biomechanical models. Three different tests were conducted at four motion analysis laboratories where clinical care and research studies are routinely performed. The laboratories had different motion capture systems, different types and number of cameras, different types and numbers of force plates and different biomechanical models. These mechanical tests evaluated the accuracy of the motion capture system, the integration of the force plate and the motion capture system, and the strength of the biomechanical model used to calculate rotational kinematics. Results of motion capture system accuracy tests showed that, for all labs, the error between the measured and calculated distances between markers was less than $2 \mathrm{~mm}$ and 1 degree for marker separations which ranged from 24 $\mathrm{mm}$ to $500 \mathrm{~mm}$. Results from the force plate integration tests demonstrated errors in center of pressure calculation of less than $4 \mathrm{~mm}$ across all labs, despite varied force plate and motion system configurations. Finally, errors across labs for single joint rotations and for combined rotations at the hip and knee were less than 2 degrees at the hip and less than 10 degrees at the knee. These results demonstrate that system accuracy and reliability can be obtained allowing the collection of comparable data across different motion analysis laboratories with varying configurations and equipment. This testing is particularly important when multi-center studies are planned in order to assure data consistency across labs.
\end{abstract}




\section{INTRODUCTION}

Three-dimensional motion analysis is commonly used to quantify the way a person moves. This technology uses camera based systems to track surface markers on the body during activities of interest and are synchronized with force plate data to calculate the intersegmental forces and moments using an inverse dynamics approach. Computational biomechanical models are then used to calculate joint kinematics and kinetics. Data derived from motion analysis studies is used to diagnose gait abnormalities, assist with patient treatment and intervention plans, evaluate the effectiveness of interventions and develop products to improve patients' quality of life, such as prosthetics. Therefore, it is essential to minimize collection error to provide reliable data.

It is important to quantify the reliability and validity of the motion analysis system and the biomechanical model used to collect and calculate joint kinematics and kinetics. Regularly conducting manufacturer calibrations ensures that equipment meets the designed specifications. However, these tests only involve a single system and equipment validation using simulated collections and simple models is also necessary to ensure the combined systems produce accurate data [1]. Accordingly, three mechanical tests were performed to ensure that varying configurations of motion capture equipment and biomechanical models produce consistent, reliable, comparable data. This is particularly important for multi-center studies in order to assure data consistency across labs.

\section{METHODS}

Four motion analysis labs conducted the proposed mechanical tests. The laboratories included in this study were the Mayo Clinic Motion Analysis Laboratory, Rochester, Minnesota and the three Centers of Excellence for Amputee Care within the Department of Defense:

Comprehensive Combat and Complex Casualty Care (C5) at the Naval Medical Center San Diego (NMCSD), California, Military Performance Laboratory, Center for the Intrepid (CFI) at Brook Army Medical Center, JBSA Fort Sam Houston, Texas, and the Center for Performance and Clinical Research Amputee Service, Walter Reed National Military Medical Center (WRNMMC), Bethesda, Maryland. Mayo, NMCSD, and CFI use Motion Analysis (Motion Analysis Corp., Santa Rosa, CA), while WRNMMC has Vicon (Vicon, Oxford, UK). At the time of testing, Mayo had 10 Raptor-12 cameras, NMCSD had 12 Eagle cameras, CFI had 10 Eagle, 8 Eagle-4, and 8 Raptor-E cameras, and WRNMMC had 26 MX cameras. Mayo had 2 AMTI force plates (AMTI, Watertown, MA; Model: BP400600) and 3 Kistler force plates (Kistler Instrument Corp., Amherst, NY; models: 9281B, 9281CA), NMCSD had 4 AMTI force plates (Model: BP600600-2000), CFI had 8 AMTI force plates (Model: OR6-7-1000), and WRNMMC had 6 AMTI force plates (Models: OR6-7, BP6001200). The anatomical models used by all the labs were the same except for the hip joint center approximation; Mayo used the Harrington model [2] while the other labs used the Bell model [3, 4]. The tracking markers were unique to each lab. 
Mayo and NMCSD used a modified Helen Hayes (HH) model [5] and CFI and WRNMMC used a modified Cleveland Clinic (CC) model [6]. The mechanical testing instrumentation was shared between labs to ensure consistency.

The Gait and Clinical Movement Analysis Society (GCMAS) Standards Council proposed motion system accuracy testing protocol, was used to track moving markers, evaluate marker locations when obscured by some cameras, and evaluate marker position when markers pass close to each other [7]. The device rotates relative to a fixed base at $120 \mathrm{rpm}$ [7]. Six markers (13 mm diameter) were mounted in fixed positions on the rotating frame and one marker (Marker7) was attached to the base, which was moved incrementally with each trial. Motion data were collected for five seconds after the rotating arm reached full speed. The distances and angles between sets of markers were calculated using the motion collection software. The reported errors for the fixed markers $\left(\mathrm{E}_{1-2}, \mathrm{E}_{3-5}\right.$, and $\left.\mathrm{E}_{345}\right)$ are the average difference between the measured and calculated distances across all trials, and marker seven errors $\left(\mathrm{E}_{7}\right)$ are for a single trial.

The method for assessing the integration of the force plates with the motion analysis system was used to give labs a simple and standardized test for evaluating and reporting system integration [8]. The integration of the force plates with the motion capture system was tested by applying a direct force to the force plate using a rod with markers attached [8]. Analog and motion data were collected at $60 \mathrm{~Hz}$ while the rod was moved in a circular pattern for three rotations. The differences between the $\mathrm{x}$ and $\mathrm{y}$ coordinates of the center of pressure of the resultant force vector and the center of the markers on the rod were calculated using custom Matlab software (The Matworks, Inc., Natick, MA).

Lastly, a physical model representing the human pelvis and right leg and foot was developed to test the accuracy of the biomechanical model. It consisted of hollow cylinders with varying radii to represent the pelvis, thigh and shank and a block to represent the foot (Fig 1). Each lab attached the markers for their specific biomechanical model to the physical model with care to avoid collinear alignment (Fig. 1A). The physical model was set to various rotational profiles (Fig. 1B), which included single rotations of \pm 10 and 20 degrees at each joint and combinations of multiple joint rotations, and manually 'walked' though the collection volume simulating heel strikes and toe-off (Fig. 1C). Visual3D (C-Motion, Inc., Germantown, MD) was used to define the anatomical coordinate system and perform the conversion from tracking marker coordinate systems to anatomical coordinate systems. Kinematic data at the hip and knee was calculated and compared to the known rotations.

\section{RESULTS and DISCUSSION}

In the marker accuracy test the labs met the minimum accuracy standards proposed by the GCMAS Standards Council (Table I) [7]. A standard was not proposed for the closest marker position so this test position was eliminated. 
In the force plate integration test, the median difference and inter-quartile range between the centers of pressure as measured by the force plate and the motion system for all force plates in each lab in the $\mathrm{x}$ and $\mathrm{y}$ directions, respectively, were for Lab HH 1: $1.98 \mathrm{~mm}(\mathrm{IQR}=1.84-2.52$ $\mathrm{mm})$ and $1.56 \mathrm{~mm}(\mathrm{IQR}=0.87-1.74 \mathrm{~mm})$; Lab HH 2: $1.12 \mathrm{~mm}(\mathrm{IQR}=0.69-1.52 \mathrm{~mm})$ and $2.46 \mathrm{~mm}(\mathrm{IQR}=1.10-3.84 \mathrm{~mm}) ; \mathrm{Lab} C \mathrm{C} 1: 2.62 \mathrm{~mm}(\mathrm{IQR}=1.88-3.54 \mathrm{~mm})$ and $3.21 \mathrm{~mm}$ $(\mathrm{IQR}=2.50-4.03 \mathrm{~mm})$; Lab CC 2: $0.48 \mathrm{~mm}(\mathrm{IQR}=0.16-1.10 \mathrm{~mm})$ and $1.38 \mathrm{~mm}(\mathrm{IQR}=1.16$ $1.84 \mathrm{~mm}$ ). These median and range values are in agreement with previously published data, which has been proposed as minimal performance criteria [8].

When comparing across sites, differences between the calculated joint angles and the physical model were greater at the knee than the hip (Fig. 2). The labs using Helen Hayes models (HH1, $\mathrm{HH} 2$ ) had greater error than the Cleveland Clinic models (CC1, CC2). This finding is similar to a previous study where the Cleveland Clinic model outperformed the Helen Hayes model for estimating tibial torsion [9]. The median difference and the inter-quartile range between the set rotation on the physical model and the biomechanical models' calculated rotations, collectively at the hip and the knee, were for Lab HH 1: 0.88 degrees (IQR=0.57 - 1.6); Lab HH 2: 1.25 degrees (IQR=0.43-4.59); Lab CC 1: 0.40 degrees (IQR=0.13-0.98); Lab CC 2: 0.11 degrees $(\mathrm{IQR}=0.11-0.98)$. The rotation values reported in this study are generally below the minimal detectable change values reported for the sagittal plane [10]. This study does not address marker placement errors that occur during in-vivo studies.

\section{CONCLUSION}

Validation and reliability testing of data collection and data processing methods is important for data quality assurance [1]. Test results demonstrated a camera issue at one site. Immediate action was taken to reposition and refocus the cameras and update the software settings. The issue was resolved and measurement error was reduced. The results of these mechanical tests demonstrate that accurate and reliable data can be obtained in motion analysis labs with varying motion capture hardware, system configuration, and biomechanical models. Mechanical testing should be performed in all motion analysis labs to assure that quality data is being collected. This testing is particularly important when multi-center studies are planned in order to assure data consistency across labs.

\section{DISCLAIMER}

The views expressed in the article are those of the authors and do not reflect the official policy of Department of the Navy, Department of the Army, Department of Defense, or the US Government.

\section{ACKNOWLEDGEMENTS}

Funding was provided by DOD 731743-1, DOD Defense Health Programs/Center for Rehabilitative Sciences Research, Grant \# HU0001-11-1-0004

There are no commercial relationships which may lead to a conflict of interest with any of the authors. 


\section{REFERENCES}

1. DeLuzio, K.J., et al., A Procedure to Validate Three-Dimensional Motion Assessment Systems. J. Biomechanics, 1993. 26(6): p. 753-759.

2. Harrington, M.E., et al., Prediction of the hip joint centre in adults, children, and patients with cerebral palsy based on magnetic resonance imaging. Journal of Biomechanics, 2007. 40: p. 595-602.

3. Bell AL, P.D., Brand RA, Prediction of hip joint center location from external landmarks. Human Movement Science, 1989. 8: p. 3-16.

4. Bell AL, P.D., Brand RA, A comparison of the accuracy of several hip center location prediction methods. J Biomech, 1990. 23: p. 617-21.

5. Kadaba M, R.H., Wootten M, Measurement of lower extremity kinematics during level walking. Journal of Orthopedic Research, 1990. 8: p. 383-92.

6. Manal K, M.D.I., Galinat B, Stanhope S, The accuracy of estimating proximal tibial translation during natural cadence walking: bone vs. skin mounted targets. Clinical Biomechanics, 2003. 18: p. 126-31.

7. Piazza, S.J., et al., A Proposed Standard for Assessing the Marker-Location Accuracy of Video-Based Motion Analysis Systems. 12th Annual GCMAS Proceedings, Springfield, MA, 2007.

8. Holden, J.P., W.S. Selbie, and S.J. Stanhope, A proposed test to support the clinical movement analysis laboratory. Gait \& Posture 2003. 17: p. 205-213.

9. Manal, K., et al., Comparison of surface mounted markers and attachment methods in estimating tibial rotations during walking: an in vivo study. Gait \& Posture, 2000. 11(1): p. 3845 .

10. Wilken, J.M., et al., Reliability and minimal detectible change values for gait kinematics and kinetics in healthy adults. Gait \& Posture, 2012. 35: p. 301-307. 
Table I. Marker Tracking Error

FIGURE LEGENDS

Figure 1. (A) Physical model of human pelvis and right leg (B) Dial used to set pelvis position with respect to thigh position to simulate hip rotation. The same configuration is used at the thigh and shank segment to simulate knee rotation. (C) 'Walking' the physical model through the motion lab to simulate a gait trial.

Figure 2. Differences (degrees) across labs between the set rotations on the physical model and the biomechanical models' calculated rotations for (A) Single Hip Rotations (B) Single Knee Rotations (C) Hip Rotations for Combined Knee and Hip Rotations (D) Knee Rotations for Combined Knee and Hip Rotations 


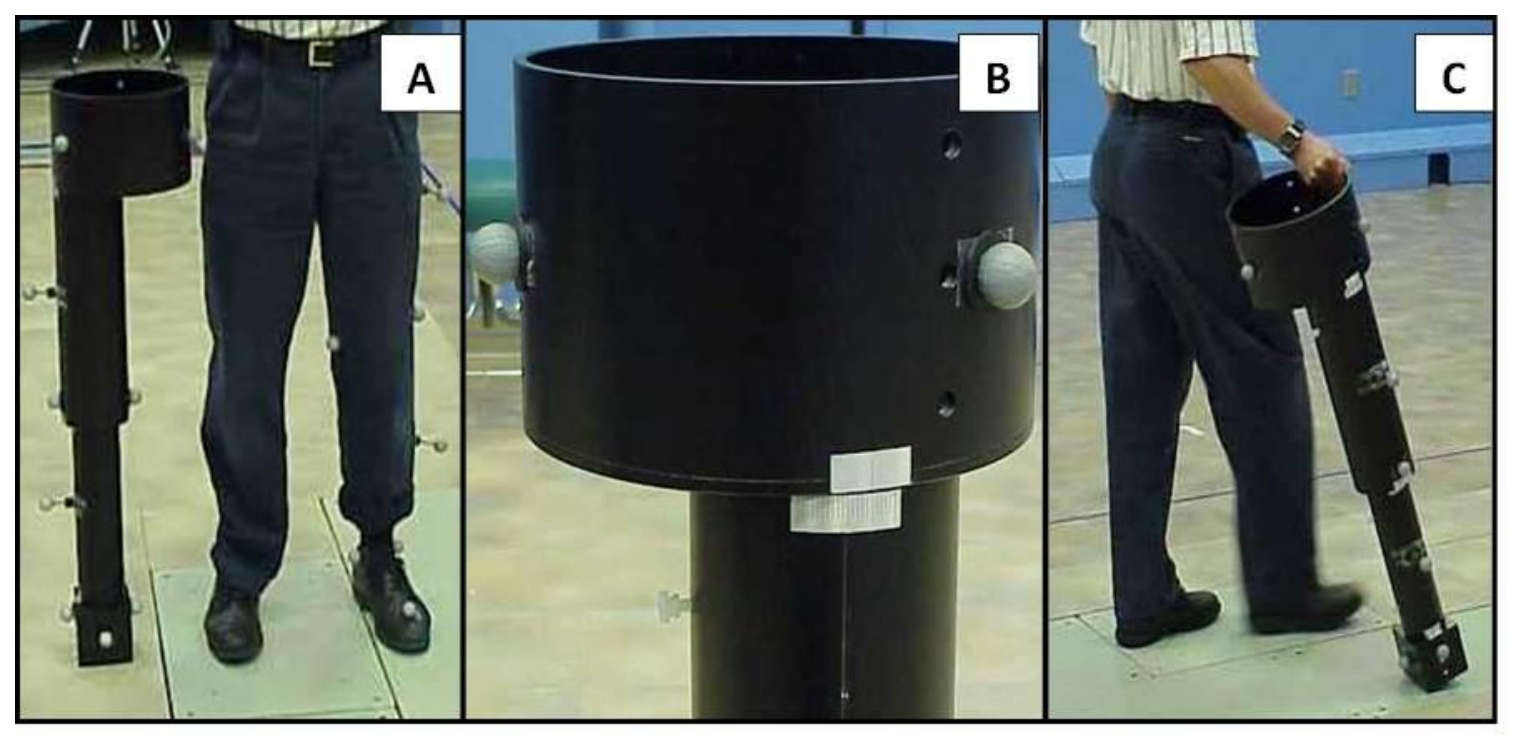




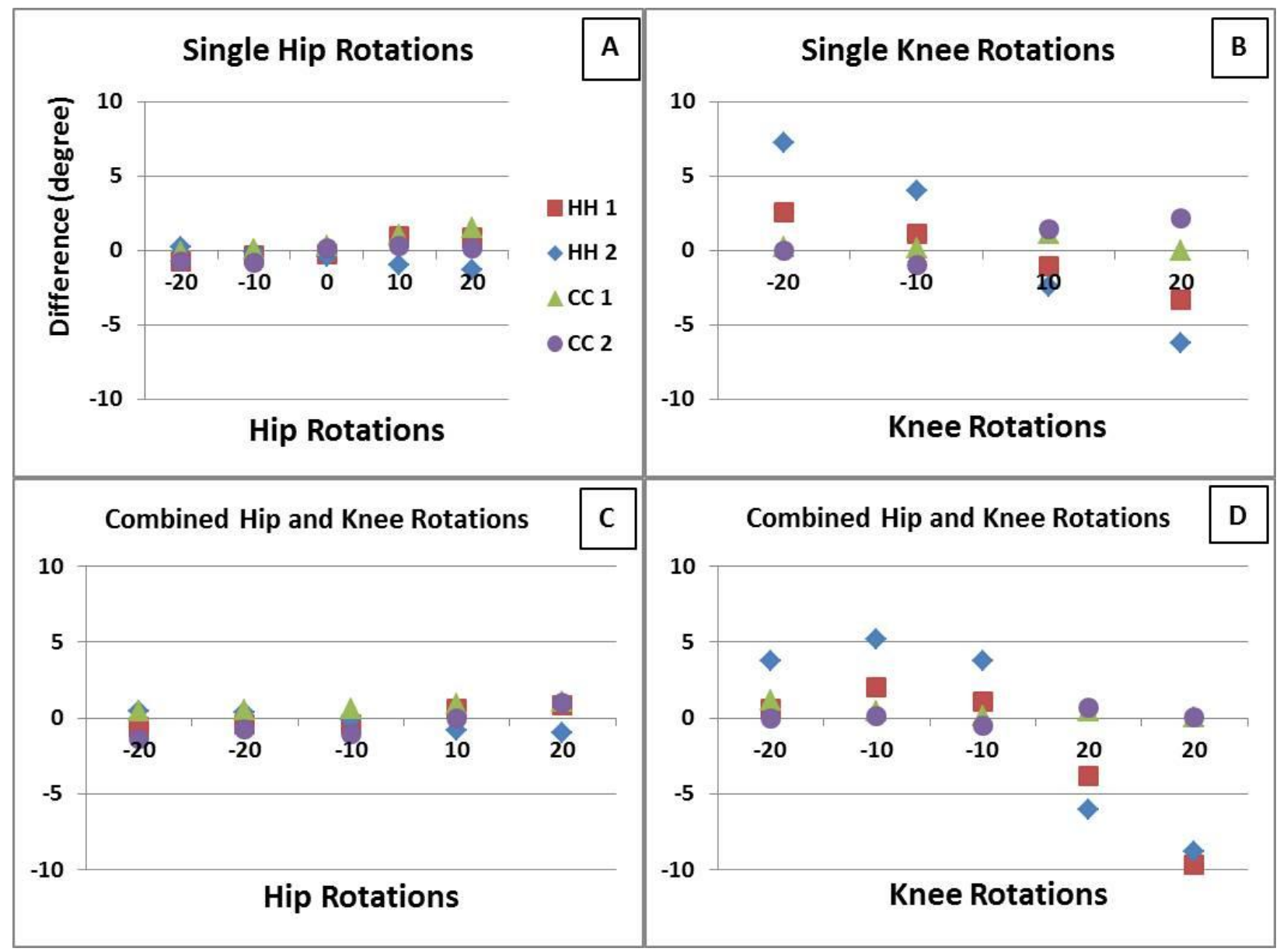




\begin{tabular}{|l|l|l|l|l|l|l|l|l|}
\hline Lab & $\begin{array}{l}\mathrm{E}_{1-2} \\
(\mathrm{~mm})\end{array}$ & $\begin{array}{l}\mathrm{E}_{3-5} \\
(\mathrm{~mm})\end{array}$ & $\begin{array}{l}\mathrm{E}_{345} \\
(\text { degree })\end{array}$ & $\begin{array}{l}\mathrm{E}_{7-5} \\
(\mathrm{~mm})\end{array}$ & $\begin{array}{l}\mathrm{E}_{7-4} \\
(\mathrm{~mm})\end{array}$ & $\begin{array}{l}\mathrm{E}_{7-3} \\
(\mathrm{~mm})\end{array}$ & $\begin{array}{l}\mathrm{E}_{7-2} \\
(\mathrm{~mm})\end{array}$ & $\begin{array}{l}\mathrm{E}_{7-1} \\
(\mathrm{~mm})\end{array}$ \\
\hline $\mathrm{HH} 1$ & 0.04 & 0.78 & 0.45 & 0.15 & 0.33 & 0.19 & 0.40 & 0.25 \\
\hline $\mathrm{HH} 2$ & 0.85 & 0.81 & 0.44 & 0.65 & 0.07 & 0.41 & 0.04 & 2.55 \\
\hline CC 1 & 1.71 & 0.46 & 0.30 & 0.46 & 0.59 & 0.59 & 0.21 & 0.48 \\
\hline CC 2 & 0.37 & 0.52 & 0.35 & 0.15 & 0.30 & 0.39 & 0.37 & 0.34 \\
\hline \hline $\begin{array}{l}\text { GCMAS } \\
\text { Standard }\end{array}$ & 1.00 & 1.00 & 1.00 & 2.00 & 2.00 & 2.00 & 2.00 & 4.00 \\
\hline
\end{tabular}

